\title{
Brighter noise: Sensory enhancement of perceived loudness by concurrent visual stimulation
}

\author{
ERIC C. ODGAARD, YOAV ARIEH, and LAWRENCE E. MARKS \\ John B. Pierce Laboratory, New Haven, Connecticut \\ and Yale University School of Medicine, New Haven, Connecticut
}

\begin{abstract}
Two experiments investigated the effect of concurrently presented light on the perceived loudness of a low-level burst of white noise. The results suggest two points. First, white noise presented with light tends to be rated as louder than noise presented alone. Second, the enhancement in loudness judgments is resistant to two experimental manipulations: varying the probability that light accompanies sound and shifting from a rating method to a forced choice comparison. Both manipulations were previously shown to eliminate a complementary noise-induced enhancement in ratings of brightness. Whereas noise-induced enhancement of brightness seems to reflect a late-stage decisional process, such as a response bias, the present results suggest that light-induced enhancement of loudness may reflect an early-stage sensory interaction.
\end{abstract}

Most studies of perceptual processes focus on vision and hearing, so it is not surprising that most studies of multimodal and cross-modal interaction focus on these two modalities. Although studies of auditory-visual interactions have a long history, three questions of broad importance are especially relevant.

1. Are, or when are, the interactions symmetrical? Are effects of visual stimulation on auditory perception accompanied by comparable effects of auditory stimulation on visual perception? It is well known that the spatial location of a visual stimulus can modify the apparent location of a simultaneously presented sound (visual capture, or "ventriloquism"; e.g., Choe, Welch, Gilford, \& Juola, 1975). Although complementary effects of an auditory stimulus on perceived visual location are unusual, these can occur under special circumstances (e.g., Radeau \& Bertelson, 1987; Warren, Welch, \& McCarthy, 1981).

2 . Where in perceptual processing do the interactions arise-for instance, at a relatively low sensory level, or at a relatively high, more cognitive, and perhaps decisional, level? Considerable evidence suggests that visual capture of auditory spatial location reflects perceptual rather than decisional interactions (e.g., Bushara et al., 2003; Driver, 1996).

3. What neural processes underlie these interactions? Although there is considerable evidence of visualauditory interactions in the central nervous system (Cal-

This research was supported in part by Grants F32 DC00463-03 to E.C.O. and R01 DC02752-03 and R01 DC00271-18 to L.E.M. from the National Institute on Deafness and Other Communication Disorders. We thank the two reviewers for their excellent comments and suggestions and Nora Williams for her help in collecting the data. Correspondence concerning this article should be addressed to E. C. Odgaard, John B. Pierce Laboratory, 290 Congress Ave., New Haven, CT 06519 (e-mail: eodgaard@jbpierce.org). vert, 2001; Stein \& Meredith, 1993), the functional consequences of these interactions remain unclear. Our present topic is the interaction between vision and audition in the perception of intensity. We report here data from two experiments done in conjunction with previous work (Odgaard, Arieh, \& Marks, 2003), in which we seek to address the first two questions and then to set forth theoretical perspectives in order to explore the third.

Two recent studies have investigated visual-auditory interactions in perceiving the intensity of a stimulus. Stein, London, Wilkinson, \& Price (1996) reported that observers tended to rate near-threshold lights as brighter when presented with a concurrent low-intensity burst of white noise than they did when the noise was absent. Relying largely on their previous work that had identified neurons responsive to multiple modalities in the brainstems of various nonhuman species (see Stein \& Meredith, 1993, for a review), Stein et al. theorized that the enhancement of brightness was sensory in origin.

Subsequently, we put Stein et al.'s theory to the test with a pair of experimental manipulations (Odgaard et al., 2003), which showed that this interaction was likely the result of a later stage decisional process, or response bias. First, we showed that the extent to which participants rate a light as brighter with noise than without depends on the relative probability of noise. Specifically, the cross-modal interaction disappeared when the probability of concurrent noise was reduced from .50 to .25 , a pattern of data consistent with psychophysical demonstrations of response bias across a broad range of tasks (e.g., Hansen \& Well, 1984; Tanner, Haller, \& Atkinson, 1967). Second, we instructed participants to make direct comparisons of brightness in a two-alternative forced choice design (Green \& Swets, 1966) and to ignore the noise but to choose the interval with the brighter light. On trials where luminance levels were equal, observers chose the interval 
without the noise as often as the interval with the noise. They were again able to ignore the effect of the noise on trials where the luminance level of the light-alone trial was increased to subjectively match the ratings enhancement of the light + noise trials from the first experiment. That is, observers tended to choose the interval without the noise, which was physically more luminous, although matched in perceived intensity to the light + noise trials.

In the two experiments reported here, we explored the converse relationship between visual and auditory signals by asking two questions. First, does concurrent light influence the loudness of low-level white noise? Second, if it does, is the interaction better explained in terms of a relatively early sensory process or a relatively later decisional process, perhaps a response bias? We addressed these questions using the same two experimental manipulations used by Odgaard et al. (2003): In one experiment, we varied the probability that a flash of light accompanied the target burst of noise, whose loudness the observers rated by setting the position of a dial (as in Odgaard et al., 2003 and Stein et al., 1996), and in another the observers compared loudness in a two-interval forced choice, where a light accompanied the noise in one of the intervals. Interestingly, the present pattern of results differs markedly from that of our earlier study, suggesting that different mechanisms may underlie the effects of light on perceived loudness and of sound on perceived brightness.

\section{EXPERIMENT 1}

Experiment 1 serves two purposes. First, it establishes whether a concurrent light affects the perception of loudness of an auditory target. Second, in the event of a positive result, this experiment determines whether the observed size of the effect depends on the probability that a light accompanies the noise. It relies on the premise that sensory processes are far less susceptible to response biases than are decisional processes. That is, if sensory interaction reflects an early-stage process, then the perception of the stimulus event, including its intensity, should be largely unaffected by the proportion of stimulus presentations that is bimodal rather than unimodal. As long as the stimuli are separated sufficiently in time to make sensory adaptation or fatigue unlikely, sensory interactions should remain fairly stable over presentation probability of the light.

Response biases, on the other hand, are highly susceptible to manipulation of presentation probability, which often exerts a systematic effect: The more frequent the biasing event, the greater the bias (e.g., Hansen \& Well, 1984; LaBerge \& Tweedy, 1964; Odgaard et al., 2003; Tanner et al., 1967). Thus, if the magnitude of the effect on loudness increases with increasing probability of light, we would interpret this as likely the result of a decisional process. The absence of an effect of probability of light, however, would be more consistent with a sensory interaction.

\section{Method}

Participants. The participants were 2 female students and 4 male students (age $M=27.2$ years, $S D=5.0$ ) at Yale University who were paid a total of $\$ 30$ for three 1-h sessions. All reported normal hearing and produced thresholds for broadband white noise at or below $18 \mathrm{~dB}$, defined as $79 \%$ correct in a two-alternative forced choice (2AFC) adaptive procedure.

Materials. The materials were the same as those used in the earlier study (Odgaard et al., 2003). The experiment was conducted in a sound-attenuating chamber (thereby occluding background luminance and ambient noise), with a chinrest mounted in place to keep the participant's head positioned 18 in. in front of the LED/speaker array. The LED was mounted in a small cylinder behind a 3.0 density neutral Wratten gelatin filter (no. 96). Its luminance was $3.2 \times$ $10^{-2} \mathrm{fL}$, as measured with a Model 2000 telephotometer from Gamma Scientific, Inc., using a $1^{\circ}$ aperture positioned at the chinrest. A pulse of broadband noise, $20 \mathrm{~Hz}-20 \mathrm{kHz}$, was produced by a signal from a Tucker Davis Technologies System 3, fed to an Optimus Pro 7AV loudspeaker positioned directly behind the LED mounting.

In our earlier study (Odgaard et al., 2003), target stimuli were spaced by approximately $0.4 \log$ units of intensity. We therefore selected five levels of noise spaced similarly, at 33, 37, 41, 45, and $49 \mathrm{~dB}(\mathrm{~A})$ overall levels, measured at the chinrest with a GenRad Model 1987 Minical sound-level meter. Duration of both the light and sound were $40 \mathrm{msec}$, with $5 \mathrm{msec}$ rise and decay for the noise. Following both Stein et al. (1996) and Odgaard et al. (2003), loudness was rated by rotating an unmarked dial $\left(340^{\circ}\right.$ turn radius), which was attached to a linear potentiometer and positioned for the participant's dominant hand. Stimulus presentation and data collection were automated in a Matlab program, operating on a PC with a $700-\mathrm{MHz}$ Pentium III processor. Instructions signaling the end of each trial and the end of each block of trials were delivered through a second loudspeaker, positioned $3 \mathrm{ft}$ to the right and below the LED mounting.

Procedure. The participants were first dark adapted with red utility goggles for $15 \mathrm{~min}$. After entering the test chamber, the participants were instructed that they would be presented with a series of trials containing a brief white noise, and that their task was to "rate the intensity of the noise by turning the dial clockwise, such that the further you turn the dial, the louder you judge that noise to be." The participants were also encouraged to use at least $70 \%$ of the dial in making their judgments. To prevent the possibility that they would avoid any effect of the light by averting their vision, the participants were then told to look for a brief flash of light accompanying some of the noises, but were informed that the presence or absence of the light was not correlated with the loudness of the noise, and that they should therefore not allow the light to influence their ratings. The participants had $3 \mathrm{sec}$ to rate each noise, at which time the computer recorded the position of the dial and then presented an auditory message instructing the participants to "reset the dial" to the maximum counterclockwise position to begin the next trial.

Each participant served in all three conditions of the experiment, which differed only in the percentages of trials containing the light - namely, $75 \%, 50 \%$, and $25 \%$. Each condition consisted of a practice block of 15 trials, after which the participant received feedback regarding the percentage of the dial used and a reminder to ignore the light when judging the intensity of the noise. Practice trials preceded three blocks of 100 test trials each, with 5-min breaks between blocks. Each of the 6 participants completed the three sessions in one of the six possible orders of presentation.

Data treatment. The recorded ratings of the linear potentiometer ranged from 0 to 10 . Analyses of the raw ratings of individual participants revealed that some, but not all, of the ratings within individual blocks were skewed beyond |.80|, and that individual blocks of trials, even within participants, had very different ranges (based on means and standard deviations). Data in blocks with 
skewed distributions were corrected either by computing the square root of each value for the blocks with positive skewness (approximately $30 \%$ of all blocks) or by squaring the values in the blocks with negative skewness (approximately $10 \%$ of all blocks). Each correction reduced the skewness below $|.80|$. Ratings from each block were then transformed to $z$ scores to remove the biasing effects of mean differences.

\section{Results and Discussion}

Figure 1 shows the average of the mean $z$-transformed ratings of loudness for each participant with repeated measures confidence intervals calculated at $95 \%$ (see Loftus \& Masson, 1994), plotted as a function of sound level. Each panel gives the results for one of the three probabilities of concurrent light. Two features of these results are noteworthy: First, for each probability, a given sound was rated louder when accompanied by light than when presented alone. Second, the enhancement in loudness ratings is more or less uniform across the three presentation probabilities.

To evaluate the results statistically, a repeated measures factorial analysis of variance (ANOVA) was performed on the averaged $z$-scored ratings across five different $\mathrm{dB}$ levels for presence/absence of light at the three different presentation probabilities. The reliable main effect of level reflects the expected result that ratings increase monotonically with stimulus intensity $[F(4,20)=$ $\left.162.74, M S_{\mathrm{e}}=0.17, p<.01\right]$. The main effect of presentation probability is uninteresting, reflecting the tolerably small amount of skewness remaining in distributions across conditions $\left[F(2,10)=65.67, M S_{\mathrm{e}}=0.01\right.$, $p<.01]$ (mean ratings were $-.07,-.05$, and $.07 z$ for the $75 \%, 50 \%$, and $25 \%$ conditions, respectively). More important was the reliable tendency for participants to rate the noise as louder when the light was present $(M=$ $.13)$ than when it was not $(M=-.14) ;[F(1,5)=75.83$, $\left.M S_{\mathrm{e}}=0.04, p<.01\right]$.

Critical to the question of whether this enhancement is sensory or decisional, however, are the interaction terms.
There was no reliable interaction between presentation probability and presence/absence of light $(F<1, p=$ .59 ), indicating that the size of the enhancement was independent of the proportion of trials containing light. Nor was there a reliable interaction among presentation probability, presence/absence of light, and dB level $(F<1, p=$ $.80)$. There was a reliable interaction between the presence/absence of light and dB level $\left[F(4,20)=3.20, M S_{\mathrm{e}}=\right.$ $0.02, p=.04]$. But this is the theoretically uninteresting result of a single unusually large difference at one level: the average $z$-score enhancement related to the presence of light ranged from .22 to .25 for all levels except $45 \mathrm{~dB}$, where the enhancement was .40. Finally, the interaction between $\mathrm{dB}$ level and presentation probability was not statistically significant $(F<1, p=.84)$.

We next calculated the average effect size for light in the three conditions, in two ways. First, we calculated the average $z$-score shift in rating in the three conditions, obtaining . 30 for the $75 \%$ condition, .25 for the $50 \%$ condition, and .24 for the $25 \%$ condition. We then calculated a partial correlation between rating and the presence/absence of light controlling for $\mathrm{dB}$ level, for each participant in each condition. We then applied meta-analytic techniques (Fisher's $z$-transformation, aggregation, and an inverse Fisher's $z$ ) to obtain an average $r$-type effect size estimate with $95 \%$ confidence intervals for each condition. This yielded a range of .17-.31 for the $75 \%$ condition, .12-.32 for the 50\% condition, and .11-.27 for the $25 \%$ condition. That all three confidence intervals overlap, and that none of them encompasses a value of zero, shows that the average effect of light is reliable for all conditions but not reliably different across them.

These data clearly show cross-modal enhancement, in that participants on average rated the noise as louder when presented with light than it was when presented alone. This general pattern holds across dB levels of noise and probabilities of light. More critically, there is no clear evidence that this enhancement occurs as the re-

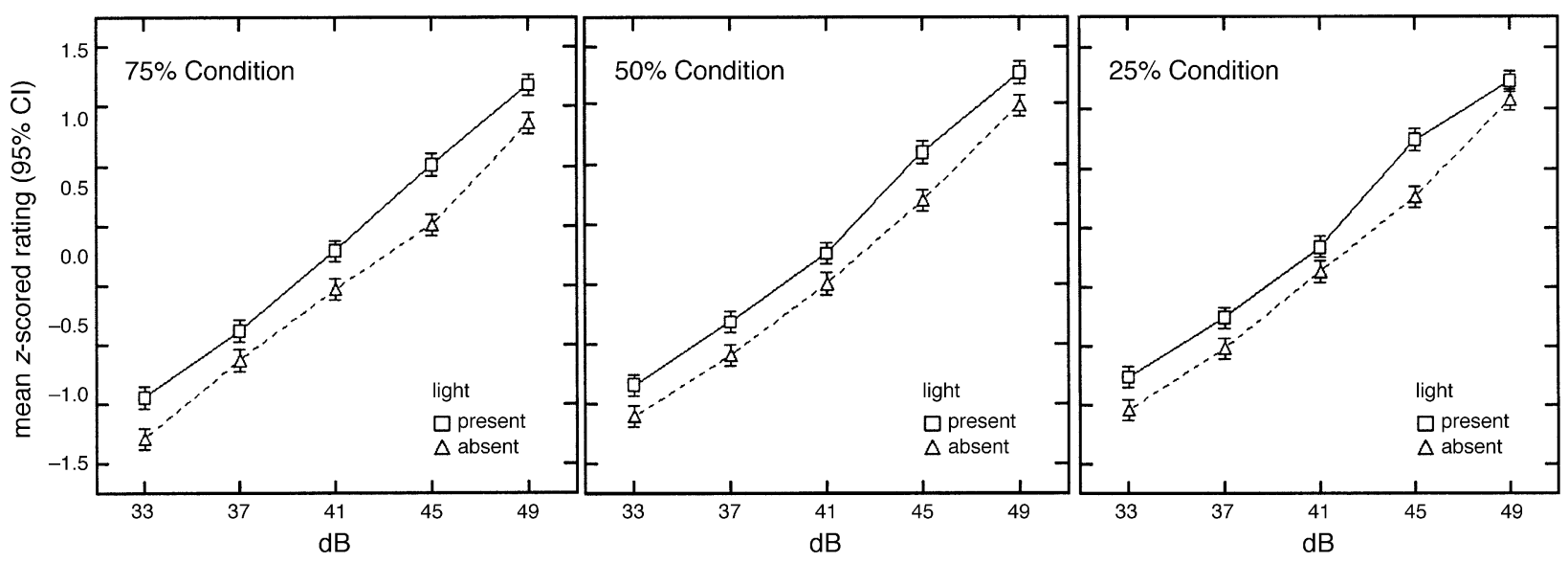

Figure 1. Average $z$ scores of intensity ratings of noise at five different dB levels, with and without concurrent light, at three different probabilities of presentation of the light. 
sult of a later stage decisional process. Consistent with sensory interaction, the magnitude of the enhancement remained relatively constant across presentation probabilities. To be sure, there may be some response biases operating here, as is common in human judgment, but there is no evidence that biases produced the substantial light-induced enhancement in loudness ratings. Instead, these results suggest the presence of sensory interaction occurring at a relatively early stage of processing.

\section{EXPERIMENT 2}

Following Odgaard et al. (2003), in Experiment 2 we sought to minimize any possible response bias by having observers make direct comparisons of loudness in a 2AFC: On each trial, the participant heard two pulses of noise, one accompanied by a light, and indicated which was louder.

In this paradigm, it does not suffice, of course, to present the noise at the same $\mathrm{dB}$ levels in both intervals of every trial. If we did so, observers might simply tend to select the interval containing the light. Consequently, as in our earlier study (Odgaard et al., 2003), we varied the levels in the two intervals according to a full factorial design. Further (as in Odgaard et al., 2003) Experiment 2 contained two conditions. In the first condition, equal $\mathrm{dB}$ $(\mathrm{EdB})$, the same five $\mathrm{dB}$ levels were used both when the noise was presented with light and when it was presented alone. If light enhances loudness, then, on average, the light-accompanied noises would be louder in EdB. In a second condition, matched loudness (ML), we increased the $\mathrm{dB}$ levels of the noise presented alone to compensate for the possible enhancement by light. In ML, the lightaccompanied noises and light-unaccompanied noises should seem, on average, equally loud.

The sensory and decisional hypotheses make different predictions. If enhancement is decisional, then either it should disappear in the forced choice design or, if observers were nevertheless biased to select intervals containing light + noise, enhancement should differ in size in the EdB and ML conditions. If enhancement is sensory, however, its size should be similar in both conditions.

\section{Method}

Participants. A second group of participants was recruited for Experiment 2. It consisted of 8 female students and 2 male students (age $M=23.2$ years, $S D=4.0$ ) at Yale University, each of whom completed two 40-min sessions as described below. They were paid $\$ 20$ for participating.

Materials. Materials and stimuli in the EdB condition mimicked those of Experiment 1: five levels of white noise, with and without concurrent light. The ML condition required a second set of white noise levels, raised in $\mathrm{dB}$ to offset the presumed enhancement by concurrent light observed in Experiment 1. We calculated these levels by dividing the average $z$-score shift resulting from the presence of light (.25) by the average $z$-score shift between $\mathrm{dB}$ levels (.59) in the $50 \%$ probability condition of Experiment 1 . The result, $42 \%$ of a 4-dB change in level, equals $1.7 \mathrm{~dB}$, so, in the ML condition, we increased the noise level presented in intervals without light by $1.7 \mathrm{~dB}$ (to $34.7,38.7,42.7,46.7$, and $50.7 \mathrm{~dB}[\mathrm{~A}]$, respectively).
Procedure. Again, the design followed Odgaard et al. (2003), except that the target was the auditory stimulus. The participants first dark adapted for 15 min with red goggles, after which we measured auditory thresholds as described earlier. All the participants gave thresholds below the lowest level used in the main experiment.

Following threshold measurement, the participants returned to the booth and received further instructions. On each trial, the participants heard two noises in succession, separated by a brief delay; the task was to decide whether the first or second of them was louder by pressing key " 1 " or " 2 " on a keyboard. The participants were informed that a brief flash of light would accompany one of the two noises in each pair, but that they should ignore the light when making their judgments. All the participants completed both conditions, in counterbalanced order.

Combining each of the five possible $\mathrm{dB}$ levels with and without light produced 25 possible pairs, and since the noise + light could appear first or second in a trial, the entire ensemble of stimulus pairs numbered 50 in each condition. In each condition, the 50 trials were presented in random order within a block, with the session for each condition containing seven such blocks, or 350 trials in all. The first block for all the participants in each condition was treated as practice and discarded before analysis. Each trial began with a 1 -sec delay, followed by the two 40 -msec stimuli, separated by $750 \mathrm{msec}$. A 1-sec delay followed the second stimulus, after which the participants were cued to enter their responses (without time constraint) before the next trial began.

\section{Results and Discussion}

For each condition, trials were parsed into two categories: (1) trials on the "diagonal" of the factorial design, in which the $\mathrm{dB}$ levels were either equal (EdB condition) or adjusted to match in loudness (ML condition), and (2) all other trials. We then calculated for each observer the proportion of trials in each of these categories on which he or she selected the interval with the light. The average proportion by condition across observers is plotted in Figure 2, along with repeated measures $95 \%$ confidence intervals (Loftus \& Masson, 1994). Note that for each bar, the statistical question is whether the observed mean is reliably different from chance (50\%) performance; these confidence intervals are therefore offered as statistical equivalents to their analogous single-sample tests.

The data present a strong picture of early-stage crossmodal interaction. The critical trials in this analysis are the "diagonals": the trials on which the dB levels were either equal (EdB) or matched in perceived loudness intensity (ML). Concurrent light affected loudness in both conditions. In the EdB condition, observers tended to choose the interval with the light as being louder, although $\mathrm{dB}$ levels were identical. In the ML condition, the response proportions were about the same, despite an actual difference of $1.7 \mathrm{~dB}$ between the levels, implying that the light-induced enhancement offset the difference in $\mathrm{dB}$. As in Experiment 1, there is some evidence of a response bias in addition to the enhancement: Observers showed a small tendency to choose the interval with the light in the "off-diagonal" trials of the EdB condition, where the levels differed by at least $4 \mathrm{~dB}$ on each trial. Because this effect is small relative to the effect in the "on-diagonal" trials and is absent from the ML condi- 


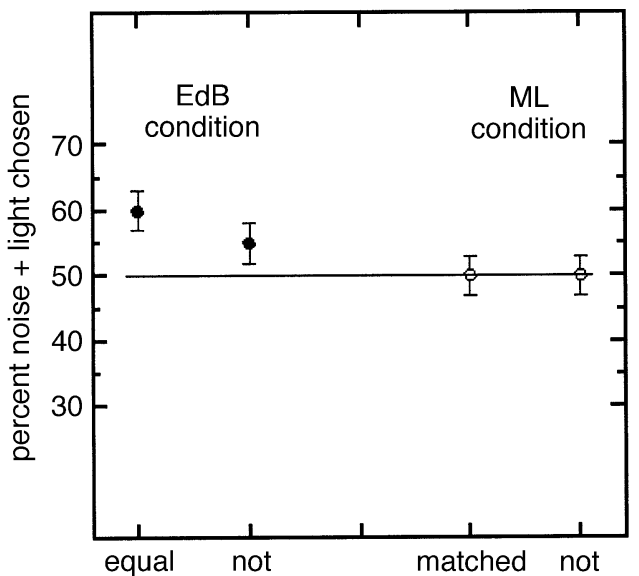

Figure 2. Proportion of intervals containing noise + light chosen as having greater sound intensity, separately for intervals in which sound levels were identical or not, in conditions in which intervals with and without light were equal in $\mathrm{dB}(\mathrm{EdB})$ and matched in loudness (ML), as rated in Experiment 1.

tion, we infer that this second-order response bias accompanies a primary, sensory enhancement. Overall, these results support the conclusion that the visualauditory interaction takes place at a relatively early stage of sensory processing.

\section{GENERAL DISCUSSION AND CONCLUSIONS}

In this study, we have asked two questions: Does concurrent light enhance the loudness of a noise? And if so, is the enhancement better explained in terms of early sensory interaction or a later decisional process such as response bias? The results show that there is indeed an enhancement of loudness. Observers tend to judge a noise as louder when it is accompanied by a flash of light. This enhancement occurs in at least two types of tasks: intensity ratings and direct comparisons.

More strikingly, and barring the presence of a decisional process resistant to traditional psychophysical means of detection and control, the data suggest that the enhancement reflects a relatively early, sensory interaction. This outcome is unusual. We know of no other compelling demonstration of sensory (nondecisional) cross-modal enhancement in the perception of intensity. Our earlier, complementary study suggested that the enhancement of brightness ratings by concurrent noise reflected a decisional rather than a sensory process. Other related studies, using measures of criterion-controlled sensitivity (Bothe $\&$ Marks, 1970) or choice response time (Marks, BenArtzi, \& Lakatos, 2003) either show no systematic visualauditory interaction (in the former case) or an interaction whose basis could not be identified (in the latter). Recently, Lovelace, Stein, and Wallace (2003) showed a small improvement in auditory threshold detection in the presence of a concurrent light, although aspects of the data suggested that this enhancement might be smaller than a response bias also observed in the data. Here, we show a strong pattern of cross-modal enhancement, above and beyond secondary indicators of a concurrent response bias.

The present results differ markedly from those of Odgaard et al. (2003). Whereas our earlier study of soundinduced enhancement of brightness showed an effect on ratings that depended on presentation probability of the sound and that disappeared in a task of direct comparison, the present light-induced enhancement of loudness was independent of presentation probability of the light and appeared in both ratings and direct comparison. Concurrent noise appeared to induce a bias in judging the perceived intensity of a light, but concurrent light actually appeared to enhance the loudness of a noise. The basis for this asymmetry remains unclear.

What neural mechanisms might underlie the crossmodal enhancement of perceived intensity? The present results, together with those of our earlier study (Odgaard et al., 2003), suggest that any prospective neural account must explain the sensory enhancement of loudness by concurrent light but the lack of analogous sensory enhancement of brightness by sound.

Studies using neuroimaging and single-cell recording techniques have identified several areas of the brain in which neurons respond to stimuli from multiple sensory modalities, especially vision and hearing (e.g., Calvert, 2001; Fort, Delpuech, Pemier, \& Giard, 2002). Many multisensory neurons seem to be involved in stimulus localization (Meredith, 2002; Stein \& Meredith, 1993), perhaps providing a mechanism for recognizing when auditory and visual stimuli arise from the same environmental source. Although we know of no evidence that multisensory neurons identified so far are involved in the perception of intensity per se, it is nevertheless possible that they play such a role.

In the present study, as in its antecedents (Odgaard et al., 2003; Stein et al., 1996), we sought to uncover interactions using relatively weak and transient stimuli. All of the noise bursts were brief $(40 \mathrm{msec})$ and relatively soft $(<50 \mathrm{~dB})$. Under such conditions, it is possible that the addition of a small input from visual activation of multisensory neurons is sufficient to augment responses to sound (Stein \& Meredith's [1993] principle of inverse effectiveness). Note that such a modest contribution should have little or no effect on responses to stronger auditory stimuli-implying that enhancement should diminish, and perhaps even disappear, when stimulus intensities increase.

Although this account could explain the sensory enhancement of loudness by light, it fails to explain the absence of sensory enhancement of brightness by sound (Odgaard et al., 2003). Why should essentially the same stimuli produce response bias when observers judge the light but sensory enhancement when they judge the noise? First, it is likely that the effects of light on loudness reflect 
inputs from multisensory neurons in the auditory system, whereas the effects of sound on brightness reflect inputs from multisensory neurons located either in the visual system or in a high-level, amodal system that mediates decision and judgment. Given the evidence for auditory input into the visual cortex (e.g., Falchier, Clavagnier, Barone, \& Kennedy, 2002), however, we are still left with the need to explain why multisensory neurons in the auditory system produce sensory enhancement in loudness, whereas multisensory neurons in the visual system do not produce a sensory enhancement in brightness. Perhaps the answer is related to the different roles that loudness plays in auditory perception and that brightness plays in visual perception.

Loudness is a basic property of most, if not all, auditory percepts. By contrast, the domain of brightness in visual perception is much more circumscribed. Brightness is a dimension that characterizes luminous objects, ranging from dim to bright. Most visual objects, however, are not luminous but contain surfaces, characterized by their lightness, which ranges from dark to light. Lightness is closely related to reflectance, and hence to the relative rather than the absolute amount of light reaching the eye from a given surface. If one of the roles of multisensory neurons is to improve the detectability of objects and events in the natural world through sensory enhancement, then auditory enhancement may occur in the perception of the lightness of visual surfaces rather than the brightness of lights. In this regard, it would be important to determine whether multisensory neurons are found in the vicinity of neurons sensitive to spatial contrast, given that contrast-sensitive mechanisms may be at least partly responsible for segregating the visual field into objects defined by their surface lightness.

\section{REFERENCES}

Bothe, G. G., \& Marks, L. E. (1970). Absolute sensitivity to white noise under auxiliary visual stimulation. Perception \& Psychophysics, 8, 176-178.

Bushara, K. O., Hanakawa, T., Immisch, I., Toma, K., KansaKu, K., \& Hallet, M. (2003). Neural correlates of multimodal binding. $\mathrm{Na}$ ture Reviews Neuroscience, 6, 190-195.

Calvert, G. (2001). Crossmodal processing in the human brain: In- sights from functional neuroimaging studies. Cerebral Cortex, 11, 1110-1123.

Choe, C. S., Welch, R. B., Gilford, R. M., \& Juola, J. F. (1975). The "ventriloquist effect": Visual dominance or response bias? Perception \& Psychophysics, 18, 55-60.

DRIVER, J. (1996). Enhancement of selective listening by illusory mislocation of speech sounds due to lip-reading. Nature, 381, 66-68.

Falchier, A., Clavagnier, S., Barone, P., \& Kennedy, H. (2002). Anatomical evidence of multimodal integration in primate striate cortex. Journal of Neuroscience, 22, 5749-5759.

Fort, A., Delpuech, C., Pemier, J., \& Giard, M. H. (2002). Early auditory-visual interactions in human cortex during nonredundant target identification. Cognitive Brain Research, 14, 20-30.

Green, D. M., \& Swets, J. A. (1966). Signal detection theory and psychophysics. New York: Wiley.

HANSEN, R. S., \& WELL, A. D. (1984). The effects of stimulus sequence and probability on perceptual processing. Perception \& Psychophysics, 35, 137-143.

LaBerge, D., \& Tweedy, J. R. (1964). Presentation probability and choice time. Journal of Experimental Psychology, 68, 477-481.

LofTus, G. R., \& MAsson, M. E. J. (1994). Using confidence intervals in within-subjects designs. Psychonomic Bulletin \& Review, 1, 476-490.

Lovelace, C. T., Stein, B. E., \& Wallace, M. T. (2003). A n irrelevant light enhances auditory detection in humans: A psychophysical analysis of multisensory integration in stimulus detection. Cognitive Brain Research, 17, 447-453.

MARKS, L. E., BEN-ARTZI, E., \& LAKatos, S. (2003). Multimodal interactions in auditory and visual discrimination. International Journal of Psychophysiology, 50, 125-145.

MEREDITH, M. A. (2002). On the neural basis for multisensory convergence: A brief overview. Cognitive Brain Research, 14, 31-40.

OdgaArd, E. C., Arieh, Y., \& MarKs, L. E. (2003). Cross-modal enhancement of perceived brightness: Sensory interaction versus response bias. Perception \& Psychophysics, 65, 123-132.

RADEAU, M., \& BERTELSON, P. (1987). Auditory-visual interaction and the timing of inputs: Thomas (1941) revisited. Psychological Research, 49, 17-22.

Stein, B. E., LONDON, N., Wilkinson, L. K., \& Price, D. D. (1996). Enhancement of perceived visual intensity by auditory stimuli: A psychophysical analysis. Journal of Cognitive Neuroscience, 8, 497-506.

Stein, B. E., \& Meredith, M. A. (1993). The merging of the senses. Cambridge, MA: MIT Press.

Tanner, T. A., JR., Haller, R. W., \& Atkinson, R. C. (1967). Signal recognition as influenced by presentation schedule. Perception \& Psychophysics, 2, 349-358.

Warren, D. H., Welch, R. B., \& McCarthy, T. J. (1981). The role of visual-auditory compellingness in the ventriloquist effect: Implications for transitivity among the spatial senses. Perception \& Psychophysics, 30, 557-564.

(Manuscript received September 23, 2003; revision accepted for publication April 22, 2004.) 